\title{
Bifosfonatos y Osteonecrosis de los Maxilares. Consideraciones Sobre su Tratamiento
}

\author{
Biphosphonate and Jaws Osteonecrosis. Considerations About of Treatment \\ "Miguel Jaimes, "Henrique Duque de Miranda Chaves Netto; ",**Sergio Olate; \\ **:* Maria das Graças Alfonso Miranda Chaves; \& *José Ricardo de Albergaria Barbosa
}

JAIMES, M.; CHAVES NETTO, H. D. M.; OLATE, S.; CHAVES, M. M. G. A. \& BARBOSA, A. J. R. Bifosfonatos asociado a osteonecrosis de los maxilares. Int. J. Morphol., 26(3):681-688, 2008.

RESUMEN: Los bifosfonatos son potentes inhibidores de los osteoclastos, son drogas usadas principalmente para el tratamiento de mieloma múltiple y en metástasis óseas de tumores sólidos como el cáncer de mama. En el año 2003 fue relatado el primer caso de osteonecrosis de los maxilares (ONM) asociado al uso de bifosfonatos, sin embargo aun no se ha establecido una relación causa efecto de estas drogas sobre la ONM. La incidencia en tiempos anteriores en relación al uso de bifosfonatos era muy baja, siendo que hoy día alcanza $10 \%$ o más en las patologías mencionadas tratadas con estas drogas. Este artículo tiene por objetivo alertar sobre una posible complicación en pacientes que hacen uso de bifosfonatos, a través de la revisión de la literatura y la presentación de un caso clínico.

PALABRAS CLAVE: Bifosfonatos, osteonecrosis, cancer, osteomielitis, metástasis ósea.

\section{INTRODUCCION}

Los bifosfonatos son potentes inhibidores de los osteoclastos ejerciendo una fuerte interacción con estas células, produciendo una marcada disminución de la reabsorción ósea. Se utilizan habitualmente en los pacientes con cáncer avanzado, metástasis óseas e hipercalcemia maligna, entre otras (Merigo et al., 2006, Migliorati et al., 2005). Además, individuos con ciertas patologías que afectan el sistema óseo, el proceso de aposición y reabsorción ósea también puede estar alterado (Haruyama et al., 2002).

En el año 2003 fue relatado por primera vez la relación existente entre el bifosfonatos y la ONM (Wang et al., 2003). Desde entonces se han reportado numerosos casos que describen este nuevo cuadro clínico que acomete a los maxilares (Merigo et al., 2006). Marx \& Stern (2003) relataron que los pacientes con mieloma múltiple que reciben pamidronate podrían desarrollar necrosis avascular de los maxilares, mientras otras publicaciones informaban sobre pacientes que requerían tratamiento para necrosis ósea intraoral de ocurrencia espontánea después de extracciones dentales o trauma oral (Marx \& Stern 2003, Migliorati 2003). Estos pacientes presentaron una historia de malignidad pri- maria, que incluía mieloma múltiple, carcinoma de mama y próstata, ya tratados con pamidronate intravenoso o con ácido zoledronico. Otros estudios han sugerido que dosis excesiva de bifosfonatos podría comprometer la calidad y densidad ósea (Pogrel 2004).

El presente artículo tiene por objetivo alertar sobre las posibles complicaciones en pacientes que hacen uso de bifosfonatos, a través de la revisión de la literatura y la presentación de un caso clínico.

Evolución de los bifosfonatos. A pesar de que los bifosfonatos fueron sintetizados en el siglo XIX, el conocimiento sobre sus características biológicas data desde la década de 1960. En el pasado, estos componentes fueron utilizados para múltiples propósitos industriales, como agente anti-sedimentación y para prevenir la formación de carbonato de cálcio.

En el año de 1865, el Prof. Fleisch y colaboradores observaron que el pirofosfato llevaba cristales de fosfato para el cálcio impidiendo la formación de cristales y disolución

\footnotetext{
"Departamento de Diagnostico Oral, División de Cirugía y Traumatología Oral y Maxilofacial. Facultad de Odontología de Piracicaba, Universidad Estadual de Campinas, Brasil.

${ }^{* *}$ Departamento de Odontología Integral, Facultad de Medicina, Universidad de La Frontera, Chile.

****Departamento de Clínica Odontológica, División de Patología Maxilofacial. Facultad de Odontología, Universidad Federal de Juiz de Fora, Brasil.
} 
in Vitro. En colaboración con los Drs. Francis y Rusell, mostraron que los análogos de los pirofosfatos, bifosfonatos, denominados en la época como difosfonatos, interactuaban de forma similar al fosfato de cálcio in Vitro, inhibiendo tanto la mineralización como la reabsorción ósea animal (Fleish et al., 1969) .

En 1969 fue publicado por primera vez sobre los bifosfonatos en el Sciencie and Nature. Veinte años más tarde estos elementos fueron utilizados en las drogas para disfunciones óseas (Fleish et al., 1969).

Los bifosfonatos presentan en su composición química dos átomos de fósforo unidos a un átomo de carbono (P-C-P). Esta estructura les confiere una resistencia a la hidrólisis enzimática y les permite fijarse con gran avidez a la superficie de los cristales de hidroxiapatita con una rápida y eficiente unión del fármaco a la superficie mineral ósea. $\mathrm{Su}$ acción primordial consiste en inhibir la reabsorción ósea mediante la supresión de la actividad de los osteoclastos (EIlemann et al., 1989).

En la investigación de Berenson et al., (1998) se observó que el uso de pamidronato mejoraba la supervivencia y disminuía las complicaciones óseas. Este y otros estudios llevaron a propugnar el uso de estas drogas de forma indefinida (Berenson et al., 1996, 2001). Siendo así, los bifosfonatos como análogos de pirofosfato, inhiben la liberación de calcio e inhibe el crecimiento de osteoclastos, permitiendo su uso en el control de lesiones malignas metastásicas, osteoporosis, enfermedad de piaget y osteogénesis imperfecta.

Consecuencia del uso del fármaco y factores asociados. Relatos sobre el uso de los bifosfonatos y consecuente necrosis de la mandíbula han sido descritos en la literatura. La necrosis es caracterizada por la muerte del hueso que resulta como una consecuencia natural de una variada gama de factores sistémicos y locales que comprometen la vascularización ósea (Assael, 2006; Merigo, 2006).

El primer estudio retrospectivo publicado sobre las exposiciones óseas con sintomatología dolorosa en la mandíbula y en el maxilar fue en el año 2003, los autores relacionaron el uso de bifosfonatos, Pamidronato y Zoledronato con la aparición de necrosis ósea. Desde este momento, diversos autores relataron casos semejantes (Leite, 2006).

Durie et al., (2005) publicó los resultados de una revisión realizada por la Fundación Internacional Mieloma, sobre los factores de riesgo en la osteonecrosis de los maxilares. Un total de 1.203 pacientes (904 con mieloma múltiple y 299 con cáncer de mama) participaron en la revisión.
Estos autores determinaron que 62 pacientes con mieloma múltiple presentaban osteonerosis de los maxilares mientras que 54 pacientes con signos de presentar la lesión. En los pacientes con cáncer de mama 13 pacientes presentaban ONM y 23 pacientes con sospecha de presentar la lesión. Corticosteroides y talidomide estaban asociados con el desarrollo de ONM. El $81 \%$ de los casos de pacientes con manifestación de la lesión presentaba historia de problemas dentales. Este estudio reporto que el $10 \%$ de los pacientes que recibieron ácido zoledronico y el $4 \%$ de los pacientes que recibieron pamidronate desarrollaron ONM. Otro estudio retrospectivo de 4.000 pacientes que recibían tratamiento con bifosfonatos intravenoso, identifico 33 casos de osteonecrosis mandibular ( $0,83 \%$ total de incidencia), 14 pacientes con diagnóstico de cáncer de mama (incidencia de $1,2 \%)$, y 15 pacientes con tratamiento para mieloma múltiple (Incidencia de 2,8\%) (Hoff et al., 2005).

Por su parte Otolina et al., (2005) realiza una revisión restospectiva de 5 pacientes que hacían uso de bifosfonatos y que desarrollaron ONM. Todos estos pacientes también presentaban historia previa de extracción dental. Los diagnósticos de ONM se han asociado fuertemente con tratamientos odontológicos, especialmente con exodoncias. La gravedad de la enfermedad determina la necesidad de realizar diagnósticos precoces, donde el uso de exámenes de imágenes podría ser trascendente.

Chaves Netto et al., (2007) reportaron un caso clínico de ONM, posterior a la colocación de implantes dentales en una paciente que hacía uso de bifosfonato. Señalaban que la paciente padecía de artritis reumatoide por 15 años, con manifestaciones en diversas articulaciones, haciendo uso crónico de diferentes drogas para su condición, incluyendo el Actonel® (Rizedronato sódico) 35mg, una dosis por semana por 2 años. El tratamiento adoptado en este caso, fue enjuague diario con clorexidina al $0,12 \%$ y controles clínicos. Posterior a 12 meses de seguimiento, los implantes fueron retirados manualmente por el odontólogo, manteniendo el tratamiento con la clorexidina $0,12 \%$; finalmente, una prótesis total inferior permitió resolver la rehabilitación oral de la paciente.

Finalmente, en la etiopatogenia de la ONM se pueden definir 4 puntos como los mas asociados a la patología: 1) radiación, 2) presencia de tejido hipoxico, hipocelular e hipovascular, 3) trauma quirúrgico y 4) exposiciones óseas (pobre cicatrización). Cuando la ONM es asociada al uso de bifosfonatos debe existir 1) exposición del hueso necrótico, 2) uso de bifosfonato y 3 ) ausencia de radiación.

Tratamiento de la osteonecrosis. Actualmente, no existe un tratamiento efectivo y definitivo para los casos de 
Tabla I. Criterios y recomendaciones propuestas para el manejo de pacientes vinculados al uso de bifosfonatos.

\begin{tabular}{|c|c|}
\hline Estado del paciente & Criterios y recomendaciones \\
\hline Pre-tratamiento. & $\begin{array}{l}\text { 1. Cualquier procedimiento dental quirúrgico debe ser finalizado antes del tratamiento con } \\
\text { bifosfonatos. } \\
\text { 2. Examen odontológico, clínica y radiográficamente, enfatizando la necesidad de mantener } \\
\text { una buena higiene bucal. } \\
\text { 3. Dientes con mal pronóstico deben ser extraídos }\end{array}$ \\
\hline En tratamiento & $\begin{array}{l}\text { 1. Control Odontológico de } 3 \text { a } 6 \text { meses. Higenización odontológica de rutina cuidadosa, } \\
\text { evitando heridas en tejidos blandos. } \\
\text { 2. Prótesis removibles deben ser chequeadas por su potencial de producir trauma. } \\
\text { 3. Tratamiento endodóntico es preferido antes la extracción dental. } \\
\text { 4. Cirugías necesaria, con antibiótico prequirúrgico y postquirúrgico por un periodo mínimo de } \\
10 \text { días. } \\
\text { 5. Cicatrización por primera intención de todas las heridas orales incluyendo sitios de } \\
\text { exodoncia. }\end{array}$ \\
\hline Pacientes con ONM & $\begin{array}{l}\text { 1. Desbridamiento mínimo de la lesión. Accesos quirúrgicos no son recomendados. } \\
\text { 2. Dispositivos removibles intra-bucales son recomendados para proteger a la lesión de } \\
\text { futuros traumas. } \\
\text { 3. Enjuages con digluconato de clorexidina al } 0,12 \% \text { es recomendado en todo los casos. }\end{array}$ \\
\hline
\end{tabular}

osteonecrosis. Algunos responden con antibioterapia, siendo útil las penicilinas asociadas con inhibidores de la penicilinasis, conjuntamente con irrigaciones locales con clorexidina al $0,12 \%$ y desbridamiento menores de secuestros óseos. En esta técnica el hueso necrótico debe ser removido hasta el área donde el tejido óseo estuviese con presencia de vascularización (Migliorati,2005).

Otros esquema antibiótico incluye suspender el uso de bifosfonatos y remoción del secuestro óseo con el uso de antibioterapia incluyendo clindamicina por 2 semanas, amoxicilina y clavulanato por 2 semanas y penicilina $G$ benzatina hasta la regresión del cuadro clínico ( Dimitrakopoulos, 2006).

Existen también otros casos donde el tratamiento consiste en el seguimiento del paciente en ausencia de intervención quirúrgica. De esta manera, el paciente deberá hacer uso de antimicrobiano durante la fase aguda, asociado a enjuagues bucales de uso continuo intentando disminuir posibles infecciones de este tejido óseo expuesto.

La remoción del hueso necrótico puede en algunas situaciones agravar el cuadro clínico. Estudios recientes han asociado factores de crecimiento como plasma rico en plaqueta durante esta técnica con la finalidad de estimular la angiogénesis y reparación del tejido óseo local. En este procedimiento todo el hueso alveolar es removido, restando solamente el componente basal. Posteriormente es colocado el plasma rico en plaqueta y finalmente se sutura la mucosa. (Curi, 2007).

La Asociación Americana de Cirugía Oral y Maxilofacial, público en el año 2007, un artículo en relación a bifosfonatos y osteonecrosis de los maxilares. El propósito de esta publicación fue establecer criterios y proporcionar directrices al respecto. Como primer punto la AACOM describe sobre el riesgo que existe en pacientes que hacen uso de bifosfonatos de desarrollar ONM. Como segundo punto, directriz de los clínicos con respecto al diagnóstico diferencial de la ONM en pacientes con historia de tratamiento de bifosfonatos por vía intravenosa o vía oral. Por último, señalan sobre las medidas preventivas al momento de tratar un paciente que haga uso de estas drogas. El tratamiento odontológico de los pacientes que están en tratamiento con bifosfonatos o que iniciaran el uso de los mismos permitirá actuar de manera preventiva. Si el paciente manifiesta la lesión el mismo será tratado dependiendo del estado actual de la enfermedad (Tabla I).

El uso de antibióticos para tratar lesiones causadas por ONM ha tenido resultados ambiguos; el criterio clínico 
continua siendo importante. La elección del antibiótico será determinada preferiblemente por el estudio de cultivo y pruebas de sensibilidad. Se ha sugerido que la combinación de amoxicilina y metronidazol puede dar buenos resultados (Migliorati, 2003; Ruggiero, 2004).

Pacientes con ONM pueden presentar infección fúngica; en estos casos también se realizan estudios de cultivo y se opta generalmente por tratamientos con nistatina oral o micostatina en comprimidos. Microorganismos, aerobios mixtos y anaerobios han sido asociados también a esta lesión (Mehrotra, 2006). El constante proceso de remodelación ósea junto a las demandas funcionales sufridas por los tejidos óseos maxilares y mandibulares permiten que el metabolismo óseo sea eficiente y constante, lo cual permitiría también un mayor desarrollo de la ONM (Gutta,2007). En pacientes en los cuales el tratamiento conservador no ha sido eficaz se debe planificar una intervención quirúrgica para eliminar el tejido óseo necrótico (Jiménez, 2005). La terapia con oxígeno hiperbárico es empleado para la prevención y tratamiento de osteorradionecrosis del componente maxilofacial; sin embargo, su uso ha sido bastante controversial. Finalmente, cuidados preventivos en todos los pacientes que van a recibir tratamiento con bifosfonatos deben ser tomados en consideración de evitar procedimientos invasivos una vez iniciado el tratamiento con estos fármacos.

Técnica Quirúrgica en el Tratamiento de Osteonecrosis. Paciente de 70 años de edad del género femenino, raza blanca, presentaba queja de exposición ósea intrabucal en la región del reborde mandibular izquierdo con aparición a 18 meses aproximadamente. La paciente se presentó asintomática, relatando historia médica positiva para osteoporosis de 7 años, con uso de Alendronato.Al examen clínico se observaron 2 exposiciones óseas en la región del reborde mandibular izquierdo con características de necrosis (Fig. 1), movilidad dentaria grado II de los elementos dentarios anteroinferiores a excepción del elemento dental 32 que presentaba movilidad dentaria grado III. La paciente hacia uso de prótesis parcial removible bimaxilar. Radiográficamente se observo radiopacidad difusa en la región mandibular izquierda de aproximadamente $0,8 \times 0,6 \mathrm{~mm}$ de diámetro, junto con reabsorción ósea generalizada debido a enfermedad periodontal. (Fig. 2)

Posteriormente a la evaluación del caso, fue definido un tratamiento quirúrgico con eliminación da la necrosis ósea sobre anestesia local (lidocaína 2\% con epinefrina 1:100.000). Se realizó una incisión a nivel de la mucosa del reborde alveolar mandibular con dos relajantes, una localizada en la región de molares y otra en la región incisivos centrales. Fue descolado el colgajo permitiendo visualizar los dos secuestros óseos necrótico, siendo observado una significativa reabsorción ósea a nivel del incisivo lateral inferior comprometiendo el pronóstico del mismo. Los secuestros óseos fueron delimitados y destacados por sus puntos de clivaje. Seguidamente se desbrido el tejido óseo necrótico con una broca de desgaste en baja rotación y abundante irrigación con suero fisiológico hasta observar la presencia de vascularización ósea. El incisivo lateral izquierdo fue extraído debido al grado de movilidad que presentaba el mismo y al comprometimiento de la necrosis ósea que se extendía a esa región (Fig. 3). Una vez finalizado el desbridamiento quirúrgico fue irrigada la región con abundante suero fisiológico siendo realizada la sutura con hilo de Nylon 4-0. Como tratamiento farmacológico post-quirúrgico fue indicado Clindamicina de $600 \mathrm{mg}$ durante 10 días y Dipirona Sódica $500 \mathrm{mg}$ durante 2 días. También se oriento al paciente a realizar enjuagues de Digluconato de Cloexidina al 0,12 \% durante 10 días y no hacer uso de la prótesis parcial removible inferior. Radiográficamente puede observarse la región donde fue realizado la ostectomia y la cercanía con el nervio mentoniano y el nervio alveolar inferior (Fig.4). En casos

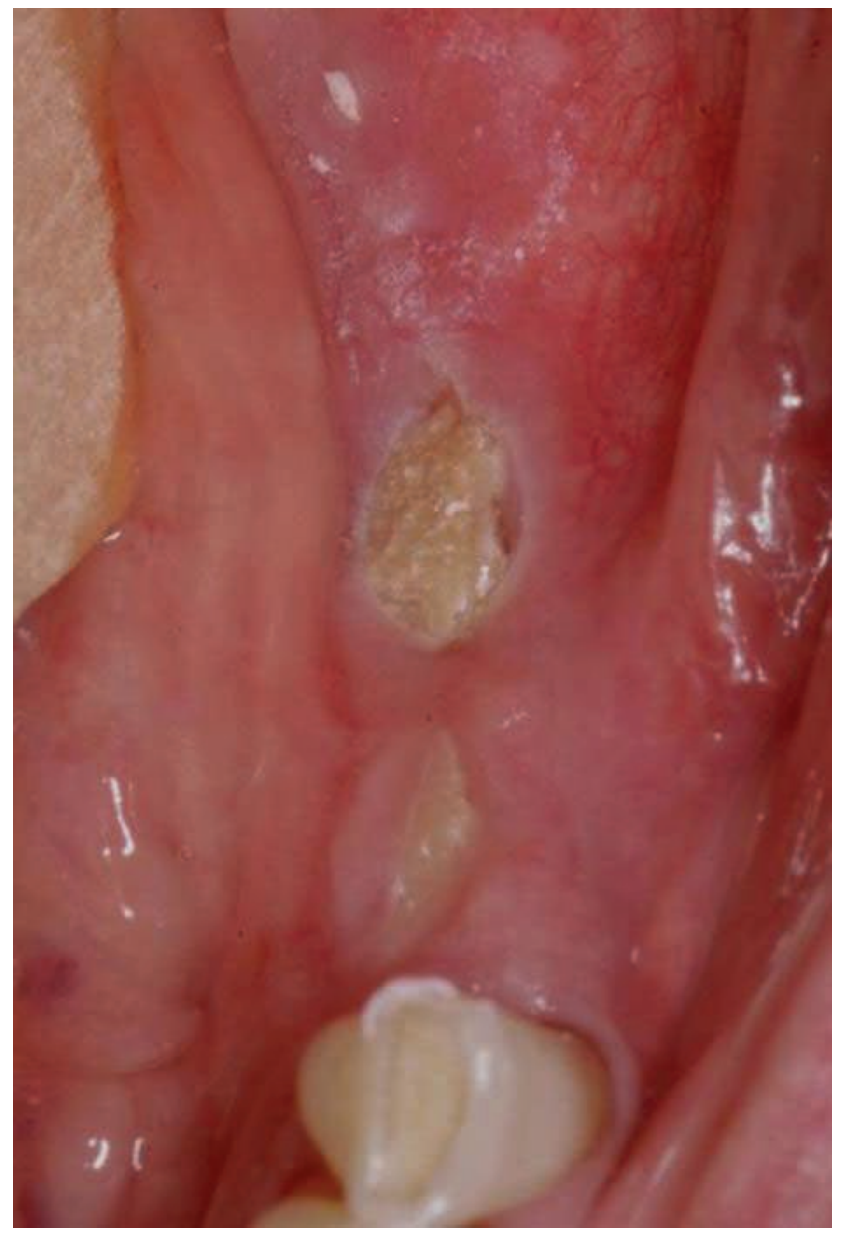

Fig. 1. Aspecto clínico de la exposición ósea en mandíbula izquierda. 


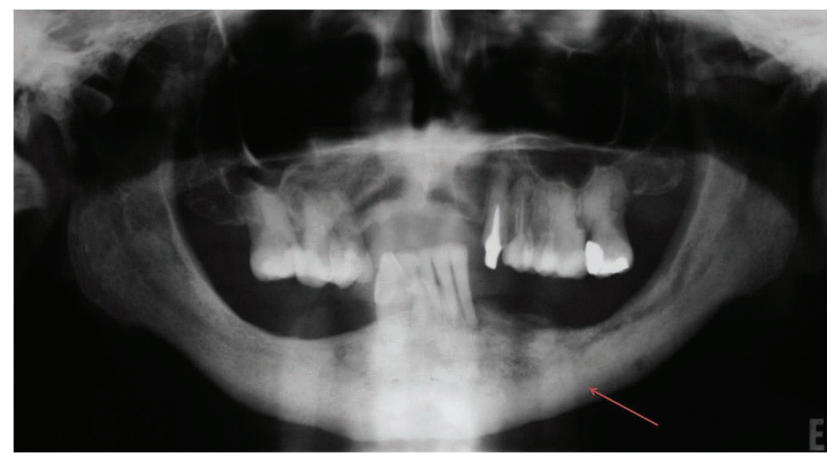

Fig. 2. Imagen radiopaca asociada al diente 3.2 y región radiopaca difusa posterior.
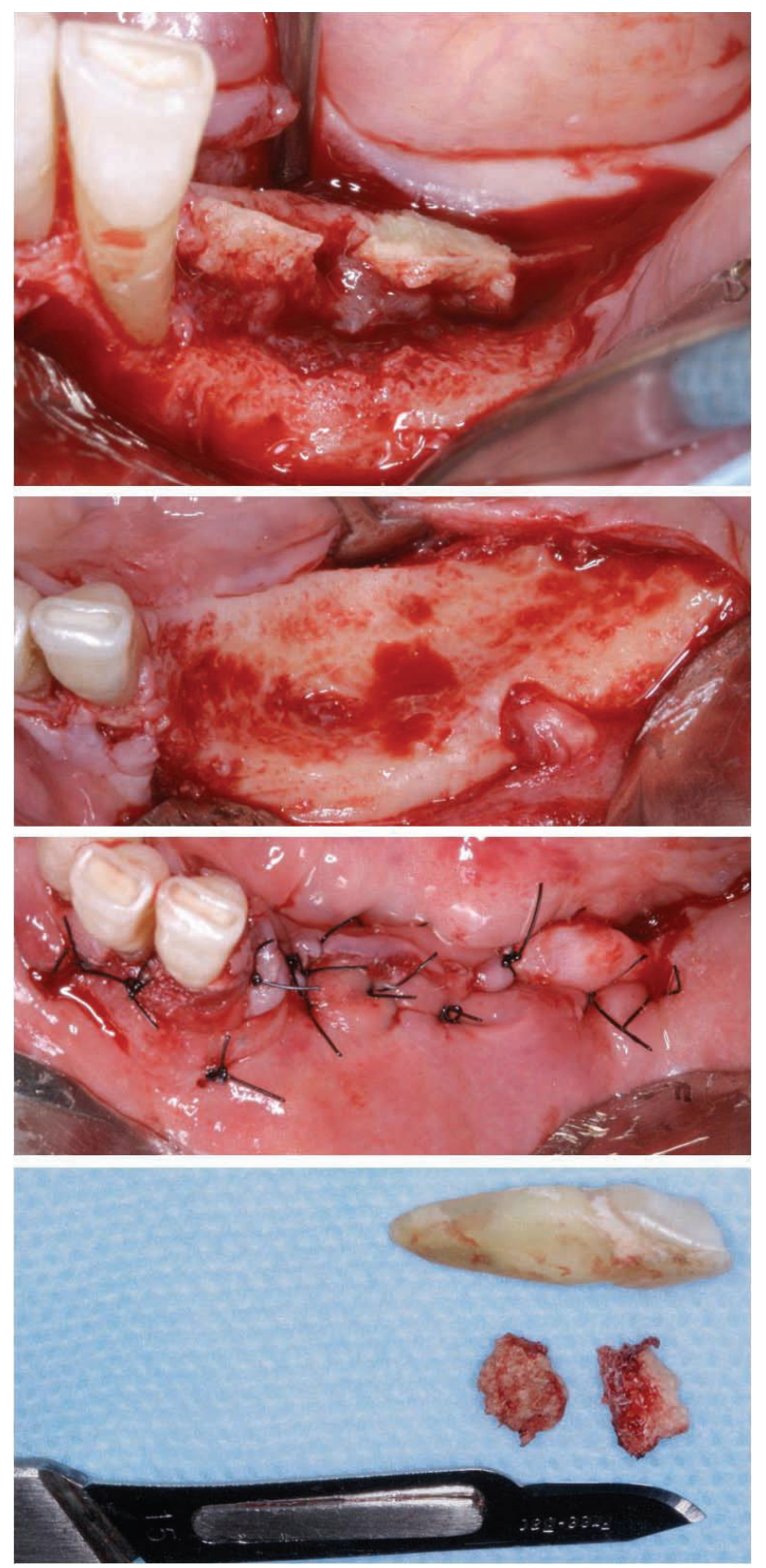

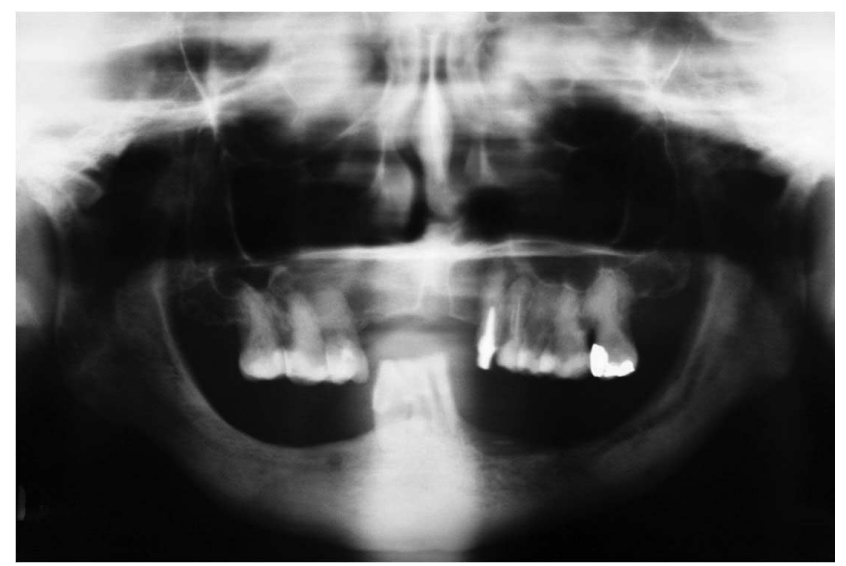

Fig. 4. Radiografía evidenciando la eliminación ósea y la exodoncia. La limitación de la ostectomia es asociada a la vitalidad ósea, sin otro parámetro clínico o radiográfico.

como este, evaluaciones permanentes en el tiempo van a contribuir para el éxito del tratamiento.

Consideraciones Finales. En el manejo de la osteonecrosis de los maxilares establecida por la Asociación Americana de Cirugía Oral y Maxilofacial, las recomendaciones son: suspensión del bifosfonato, higienización oral con clorhexidina $0,12 \%$ en áreas óseas expuestas y especialmente en la zona de inserción mucoperióstica, que es propicia a la colonización bacteriana. En caso de molestia lingual por contacto con áreas óseas o en presencia de infección secundaria persistente, puede ejecutarse ostectomías limitadas y cobertura antibiótica para evitar infección secundaria. Como ya fue señalado, el uso de oxigenoterapia en cámara hiperbárica, para mejorar la oxigenación local, no se ha demostrado uniformemente eficaz (Ruggiero, 2004).

El tiempo medio entre el primer uso de bifosfonatos y la exposición ósea necrótico depende del medicamento, variando de 9 meses a 3 años, pudiendo estar asociado a cuadros dolorosos, movilidad dentaria y fistulas (Marx, 2005).

Como explicación de la frecuente localización en los maxilares, se ha resaltado el estrés fisiológico a que son sometidas dichas áreas, únicas en exponer hueso al medio externo. Este estrés aumenta por procesos patológicos dentales y periodontales junto a su tratamiento, como las infecciones con tratamiento endodóntico, extracciones dentales y otros, que exigen aumentar la velocidad de regeneración y

Fig. 3. Técnica quirúrgica de eliminación del tejido necrótico. El colgajo debe ser extenso para permitir cicatrización por primara intención y debe eliminarse todo el tejido que no se presente vital (con sangramiento) al momento del desgaste con broca. 
remodelación ósea, la que se encuentra bloqueada por dichas drogas (Sook-Bin et al., 2005, Santini et al., 2002). A esto se agrega el efecto antiangiogénico de los bifosfonatos, que provocan disminución del factor de crecimiento endotelial (Vitte et al., 1996, Durie et al., 2005), posiblemente explicando la mayor frecuencia de compromiso de la mandíbula (63-80\%), hueso de menor irrigación en relación a la maxila (14-38\%), (Marx \& Stern 2003, Jiménez \& Bagan 2005). La mayoría de los casos de osteonecrosis se presentan posterior a una extracción dental (78\%), infección u otro proceso y sólo una minoría (14\%) aparece espontáneamente. Sólo 5,5\% se presentan en ambos maxilares (Marx \& Stern 2003, Vitte et al., 1996).

Pacientes con factores de riesgo mencionados, se han comunicado las siguientes recomendaciones en la prevención: evaluación dental previa al inicio del tratamiento con bifosfonatos, seguimiento y tratamiento de cualquier patología dentomaxilar, extracción de restos radiculares, tratamiento de la enfermedad periodontal y restauraciones, con el mismo criterio recomendado para pacientes candidatos a radioterapia (Sook-Bin et al., 2005). Higiene dental con antisépticos locales durante la terapia (Pogrel 2004). Realizar estudios de imágenes ante cualquier síntoma local (Berenson et al., 1998, Haruyama et al., 2002). Evitar cirugía dental como exodoncias, implantes, debridaciones, entre otros, durante la terapia farmacológica (Marx \& Stern 2003, Berenson et al ., 2001, Tarassoff \& Yong-jiang 2005), y suspender el uso de bifosfonatos 3 meses antes de procedimientos dentales que se consideren imprescindibles durante la terapia (Thakkar et al., 2005).

La ONM ha sido asociada en los últimos años al uso prolongado de bifosfonatos. Estas drogas deben ser usadas con precaución, especialmente en aspectos preventivos. Como se puede notar en esta investigación, hasta el momento no se tiene establecido un tratamiento efectivo para los casos de osteonecrosis. Conocer sobre las medidas preventivas recomendadas para aquellos pacientes que seran sometidos a tratamientos odontológicos y que iniciarán o están en tratamiento con bifosfonato permitirá decidir sobre la ejecución o no de ciertos tratamientos odontológicos.

JAIMES, M.; CHAVES NETTO, H. D. M.; OLATE, S.; CHAVES, M. M. G. A. \& BARBOSA, A. J. R. Biphosphonate and jaws osteonecrosis. Considerations about of treatment. Int. J. Morphol., 26(3):681-688, 2008.

SUMMARY: Bisphosphonates are strong osteoclastic inhibitor activities, being these drugs usually for treatment of multiple myeloma and bone metastases of solid tumors like breast cancer. The osteonecrosis of the maxillary bones was first described in 2003. The relation of these medicines and maxillary bone osteonecrosis still uncertain. The incidence of this bone necrosis was very low before biphosphonates using. Nowadays this complication reach at least $10 \%$ of patients in these therapy using. The present work mean to alert for possible complication in biphosphonates using patients, thru a literature review and a clinical case presentation.

KEY WORDS: Bisphosphonate, osteonecrosis, cancer, osteomielitis, bony metastasis.

\section{REFERENCIAS BIBLIOGRÁFICAS}

Advisory Task Force on Bisphosphonate-Related Ostenonecrosis of the Jaws, American Association of Oral and Maxillofacial Surgeons. American Association of Oral and Maxillofacial Surgeons position paper on bisphosphonate-related osteonecrosis of the jaws. J. Oral Maxillofac. Surg., 65(3):369-76, 2007.

Assael L. A. A Time for Perspective on Biphosphonates. J. Oral Maxillofac. Surg., 64:877-9, 2006.

Bagan, J. V.; Murillo, J.; Jimenez, Y.; Poveda, R.; Milian, M. A.; et al. Avascular jaw osteonecrosis in association with cancer chemotherapy: Series of 10 cases. J. Oral Pathol. Med., 34: 120-3, 2005.

Berenson, J. R.; Lichtenstein, A.; Porter, L.; Dimopoulos, M. A.; Bordoni, R.; George, S.; et al. Long-term pamidronate treatment of advanced multiple myeloma patients reduces skeletal events. Myeloma Aredia Study Group 1. J. Clin. Oncol., 16: 593-02, 1998.

Berenson, J.R.; Lichtenstein, A.; Porter, L.; Dimopoulos, M.A.; Bordoni, R.; George, S.; et al. Efficacy of pamidronate in reducing skeletal events in patients with advanced multiple myeloma. N. Engl. J. Med. 334: 48893, 1996.

Berenson, J.R.; Rosen, L.S.; Howell, A.; Porter, L.; Coleman, R.E.; Morley, W.; et al. Zoledronic acid reduces skeletalrelated events in patients with osteolytic metastases. Cancer 91:1191-200, 2001.

Chaves-Netto, H. D. M.; Lisbôa, R. B.; Ortega, R. L. \& Mazzonetto, R. Osteonecrose mandbular após terapia por 
implantes oseintegrados decorrente do uso de bifosfonatos. Revisão da Literatura e relato de caso. Impl. News 4(3): 27-30, 2007.

Chiandussi, S.; Biasotto, M.; Dore, F.; Cavalli, F.; Cova, M. A. \& Di Lenarda, R. Clinical and diagnostic imaging of bisphosphonate-associated osteonecrosis of the jaws. Dentomaxillofac Radiol., 35: 236-43, 2006.

Curi, M. M.; Cossolin, G., S., I.; Koga, D., H.; Araújo, S., R.; Feher O.; Santos, M., O. \& Zardetto C. Treatment of Avascular Osteonecrosis of the Mandible in Cancer Patients With a History of Bisphosphonate Therapy by Combining Bone Resection and Autologous Platelet-Rich Plasma: Report of 3 Cases. J. Oral Maxillofac. Surg., 65:349-55, 2007.

Dimitrakopoulos I.; Magopoulos C.; Karakasis D. Biphosphonates-induced avascular osteonecrosis of the jaws: a clinical report of 11 cases. Int. J. Oral Maxillofac. Surg., 35:588-93, 2006.

Durie, B. G. M.; Katz, M. \& Crowley, J. Osteonecrosis of the jaw and bisphosphonates. N. Engl. J. Med., 353: $99-100,2005$.

Eilemann, K.; Sjogren, P.; Banning, A. M. \& Jensen, D. Trial of intravenous lidocaine on painful neuropathy in cancer patients. Clin. J. Pain, 5: 291-4, 1989.

Fleish, H.; Russell, R. G. \& Francis, M. D. Diphosphonates inhibit hydroxyapatite dissolution in vitro and bone resorption in tissue culture and in vivo. Science 165:1262-4, 1969.

Fleish, H.; Russell, R. G.; Simpsom, B. \& Muhlbauer, R. C.; Prevention by diphosphonate of immobilization osteoporosis in rats. Nature, 223:211-2, 1969.

Gutta, R. \& Louis, P. J. Bisphosphonates and osteonecrosis of the jaws: Science and rationale. Oral Surg. Oral Med. Oral Pathol. Oral Radiol. Endod. 104(2):18693, 2007.

Haruyama, N.; Igarashi, K.; Saeki, S.; Otsuya-Isoya, M.; Shinoda, H. \& Mitani, H. Estrous-cycle-dependent variation in orthodontic tooth movement. J. Dent. Res., 6: 406-10, 2002.

Hoff A.O.; Toth B. \& Altundag K. Osteonecrosis of the jaw in patinents receiving intravenous bisphosphona tetherapy. American Society for boné and Mineral Research, September, 2005.
Jiménez-Soriano, Y. \& Bagan, J. V. Los bifosfonatos, nueva causa de osteonecrosis maxilar por fármacos: situación actual. Med. Oral Patol. Oral Cir. Bucal, 10(Suppl 2): E88-91, 2005.

Kumar, V.; Pass, B.; Guttenberg, S. A.; Ludlow, J.; Emery, R. W.; Tyndall, D. A. \& Padilla, R. J. Bisphosphonaterelated osteonecrosis of the jaws: A report of three cases demonstrating variability in outcomes and morbidity. J. Am. Dent. Assoc. 138(5):602-9, 2007.

Leite A.F. et al. Biphosphonates-associated osteonecrosis of the jaws. Report of a case and literature review. Oral Surg. Oral Med. Oral Pathol. Oral Radiol. Endod., 102: 14-21, 2006.

Marini, J. R. Do bisphosphonates make children's bones better or brittle? N. Engl. J. Med., 349: 423-6, 2003.

Marx, R. E. \& Stern, D. Oral and maxillo-facial pathology: a rationale for diagnosis and treatment. $1^{\mathrm{a}} \mathrm{ed}$. Quintessence, 2003. pp 36-38.

Mehrotra, B. \& Ruggiero, S. Bisphosphonate complications including osteonecrosis of the jaw. Hematology Am. Soc. Hematol. Educ. Program, 1: 356-60, 2006.

Merigo, E.; Manfredi, M.; Meleti, M.; Guidotti, R.; Ripasarti, A.; Zanzucchi, E.; et al. Bone necrosis of the jaws associated with bisphosphonate treatment: a report of twenty-nine cases. Acta Biomed., 77(2):10917, 2006.

Migliorati, C. A. Bisphosphonates and oral cavity avascular necrosis of bone. J. Clin. Oncol., 21:4253-4, 2003.

Migliorati, C. A.; Schubert, M. M.; Peterson, D. E. \& Seneda, L. M. Bisphosphonate-associated osteonecrosis of mandibular and maxillary bone. Cancer, 104(1):83-93, 2005.

Oltolina, A.; Achilli, A.; Lodi, G.; Demarosi, F. \& Sardella, A. Osteonecrosis of the jaws in patients treated with bisphosphonates. Review of the literature and the Milan experience. Minerva Stomatol., 54:441-8, 2005.

Pogrel, M. A. Bisphosphonates and bone necrosis. J. Oral Maxillofac. Surg., 62: 391-92, 2004.

Ruggiero, S. L.; Mehrotra, B.; Rosenberg, T. J. \& Engroff, S. L. Osteonecrosis of the jaws associated with the use of bisphosphonates:a review of 63 cases. J. Oral Maxillofac. Surg. 62:527-34, 2004. 
Santini, D.; Vincenzi, B.; Avvisati, G.; Dicuonzo, G.; Battistoni, F. \& Gavasci, M.; et al. Pamidronate induces modifications of circulating angiogenic factors in cancer patients. Clin. Cancer Res., 8:1080-4, 2002.

Sook-Bin, W.; Hande, K. \& Richardson, P. G. N. Eng. J. Med., 353:100, 2005.

Tarassoff, P.; Yong-Jiang, H. Reply from Novartis. N. Engl. J. Med., 353: 101-2, 2005.

Thakkar, S. G.; Isada, C.; Smith, J.; Karam, M. A.; Reed, J.; Tomford, J. W.; et al. Jaw complications associated with bisphosphonate use in patients with plasma cell dyscrasias. Med. Oncol. 23(1):51-6, 2006.

Vitte, C.; Fleisch, H. \& Guentes, H. L. Bisphosphonates induce osteoblasts to secrete an inhibitor of osteoclastic mediated resorption. Endocrinology, 137:2324-33, 1996.

Wang, J.; Goodger, N. M. \& Pogrel, M. A. Osteonecrosis of the jaws associated with cancer chemotherapy. J. Oral. Maxillofac. Surg., 61:1104-7, 2003.

Whyte, M. P.; Wenkert, D.; Clements, K. L.; McAlister, W. H. \& Mumm, S. Bisphosphonate-induced osteopetrosis. N. Engl. J. Med. 349(5):457-63, 2003.
Dirección para correspondencia: Miguel Angel Jaimes Pérez Departamento de Diagnóstico Oral División de Cirugía y Traumatología Buco-Maxilo-facial

Avenida Limeira, 901

Bairro: Areão

CEP: 13414-903

Piracicaba-SP

BRASIL

Fone: (55) 19 - 92258022

Email: miajape@fop.unicamp.br

Received: 25-03-2008

Accepted: 14-08-2008 